\title{
Transcriptional Regulation of KCS Gene by Bzip29 and MYB70 Transcription Factors in Respond to ABA-Stimulated Wound Suberization of Kiwifruit
}

\section{Xueyuan Han}

Shaoxing University

Xiaopeng Wei

Zhengzhou University of Light Industry

Wenjing Lu

Zhejiang Academy of Agricultural Sciences

Qiong Wu

Henan University of Technology

Linchun Mao ( $\nabla$ linchun@zju.edu.cn )

Zhejiang University

\section{Research Article}

Keywords: suberization, KCS, transcriptional regulation, abscisic acid

Posted Date: July 28th, 2021

DOI: https://doi.org/10.21203/rs.3.rs-712087/v1

License: (c) (i) This work is licensed under a Creative Commons Attribution 4.0 International License. Read Full License 


\section{Abstract}

Background: Our previous study has demonstrated that the transcription of AchnKCS involved in suberin biosynthesis was up-regulated by exogenous abscisic acid (ABA) during the wound suberization of kiwifruit, but the regulatory mechanism has not been fully elucidated.

Results: Through subcellular localization analysis in this work, AchnbZIP29 and AchnMYB70 transcription factors were observed to be localized in the nucleus. Yeast one-hybrid and dual-luciferase assay proved the transcriptional activation of AchnMYB70 and transcriptional suppression of AchnbZIP29 on AchnKCS promoter. Furthermore, the transcription level of AchnMYB70 was enhanced by ABA during wound suberization of kiwifruit, but AchnbZIP29 transcription was reduced by ABA.

Conclusions: Therefore, it was believed that ABA enhanced the transcriptional activation of AchnMYB70 on AchnKCS by increasing AchnMYB70 expression. On the contrary, ABA relieved the inhibitory effect of AchnbZIP29 on transcription of AchnKCS by inhibiting AchnbZIP29 expression. These results gave further insight into the molecular regulatory network of $A B A$ in wound suberization of kiwifruit.

\section{Background}

Fruits are often bruised or mechanically wounded during the harvesting, transportation and storage processes, which leads to the susceptibility to microbial infection and quality degradation. However, the damaged surface of the postharvest kiwifruit would suberize to accumulate suberin and further form a healing layer, which can reduce the outflow of cell water and nutrients and limit the invasion of pathogens [1-3]. Suberin, a kind of plant secondary metabolite, is a polymer composed of glycerol-based polyphenols and polylipids. Wounding-induced suberization also commonly occurs in potato tuber [4], Arabidopsis root [5] and postharvest tomato [6].

Exogenous abscisic acid (ABA) could stimulate the accumulation of suberin with induced expression of genes encoding $\beta$-ketoacyl-coenzyme A synthases (KCSs) related to suberin synthesis $[1,7]$. KCSs, as the components of fatty acid elongation enzyme complex (FAE), catalyze the condensation of long-chain fatty acyl CoA and malonyl CoA to produce $\beta$-ketoacyl CoA with a carbon chain extension of two-carbon unit (Fig. 1), participating in the synthesis of very long chain fatty acids (VLCFAs) that are the precursors of suberin biosynthesis.

QsMYB1 (Quercus suber) was reported to target two QsKCS involved in suberin biosynthesis by Chip-seq assay [8]. Recently, it was revealed that AchnbZIP12 responding to ABA signaling positively regulated the transcription of AchnKCS during wound suberization of kiwifruit [9]. AtMYB41 [10], AtMYB9 [11], AtMYB107 [12] and AtMYB93 $[13,14]$ were demonstrated to be associated with the regulation of suberin biosynthesis. The over expression of MYB92 in leaves of Nicotiana benthamiana significantly increased the transcript level of $K C S 1$ and the deposition of corresponding suberin monomers with carbon chain length of $>20$ [15]. Similarly, the transcript levels of KCS2 and KCS2O were elevated in MYB39 overexpression leaves of $\mathrm{N}$. benthamiana, and KCS1 and KCS2 in MYB39 overexpression root of 
Arabidopsis [16]. Moreover, some of these transcription factors involved in suberization regulation have been shown to be ABA-responsive, such as AtMYB41 [10], AchnbZIP12 [9] and AchnMYB107 [17]. Besides, ABA signaling cascades was suggested to play a mediating role in suberin biosynthesis regulated by MYB39 in the Arabidopsis root endodermis [16].

Therefore, based on our previous report and related literatures, the present study was to explore the regulatory mechanism of $A B A$ in inducing AchnKCS expression during suberin deposition by investigating the transcriptional control of transcription factors on AchnKCS. AchnbZIP29 and AchnMYB70 transcription factors were speculated and verified to regulate the transcription of AchnKCS in respond to ABA-stimulated wound suberization. It was expected to give further insight into the molecular regulatory network of $A B A$ in promoting wound suberization of kiwifruit.

\section{Methods}

\section{Fruit treatment}

Kiwifruit (Actinidia deliciosa cv. Xuxiang) were harvested at commercial maturity with the uniformity of shape and size from a commercial orchard in Fuyang District, Hangzhou, China. Treatment was based on Han et al. [9]. The surface was sterilized with $0.5 \%$ (v/v) NaClO solution for 3 min, washed with sterile water and air-dried naturally. Artificial wound was made by cutting the fruit into halves lengthwise. Fruit halves were treated respectively with $0.5 \mathrm{mmol} \mathrm{L}^{-1} \mathrm{ABA}$ ( $\geq 90 \%$, Aladdin Industrial Inc., China) and sterile water (control) by vacuum infiltration. Afterwards, fruit halves were stored in a sterile incubator at $20{ }^{\circ} \mathrm{C}$ and $85 \%$ relative humidity for wound healing under darkness. Suberized tissue was separated from the scarred outmost layer of the wound surface after incubating for 2, 3 and 4 days and stored at $-80^{\circ} \mathrm{C}$ until further analysis.

\section{RNA extraction}

The cetyltrimethylammonium bromide (CTAB) method was carried out to extract the total RNA [18]. The implementation details referred to Han et al. [9]. Briefly, 2\% CTAB extraction buffer and LiCl solution (12 mol L-1) were applied to extract and denature the RNA on the first day. On the second day, the SSTE buffer (containing $1.0 \mathrm{mM}$ EDTA, $10 \mathrm{mM}$ Tris- $\mathrm{HCl} \mathrm{pH} \mathrm{8.0,0.5 \%} \mathrm{(m/v)} \mathrm{SDS} \mathrm{and} 1.0 \mathrm{M} \mathrm{NaCl}$ ), chloroform and ethanol were added to dissolve, purify and precipitate the RNA, respectively. Finally, wash the RNA pellet with pre-chilled $75 \%$ ethanol for twice and dissolve the RNA pellets again using RNase-free water. The quality of the RNA samples was measured using a NanoDrop 2000 (Thermo Fisher Scientific, USA).

\section{DNA extraction}

The total DNA was extracted by implementing the CTAB method [19]. The implementation details referred to Han et al. [9]. Briefly, 2\% CTAB buffer, the solution of phenol: chloroform: isoamylol (25:24:1) and the solution of chloroform: isoamylol (24:1) were applied to extract and purify the DNA. After centrifuging, NaAc solution and isopropanol were added to precipitate the DNA. Afterwards, wash and dissolve the 
DNA precipitate respectively with $75 \%(\mathrm{v} / \mathrm{v})$ ethanol and TE buffer. The quality of DNA samples was measured by a NanoDrop 2000.

\section{Molecular cloning}

The gene sequence of transcription factor AchnbZIP29 (Achn340751) and AchnMYB70 (Achn117821) were identified based on the Cornell University kiwifruit database (http://bioinfo.bti.cornell.edu/cgi-bin/kiwi/home.cgi). The cloning conditions were according to Han et al. [9]. Based on the primers in Supplementary Table 1 (AchnbZIP29-Full and AchnMYB70-Full), both genes of AchnbZIP29 and AchnMYB70 were cloned from reverse transcribed cDNA. And the promoter of AchnKCS was cloned from the extracted total DNA using the corresponding AchnKCS-Pro primers. After linking the amplified product with pEASY-T1 simple vector and transferring it into Escherichia coli, the test of white spot screening was carried out to obtain the recombinant plasmid.

\section{Subcellular localization of AchnbZIP29 and AchnMYB70}

After cloning the CDS of AchnbZIP29 and AchnMYB70, the sequence with no stop codon was amplified and inserted into the 1300-35S-eGFP vector. The obtained AchnbZIP29-GFP and AchnMYB70-GFP fusion expression vectors were respectively transferred into Agrobacterium strain. The preparation of the infection buffer of Agrobacteria and the inoculation of tobacco (Nicotiana benthamiana) leaves were according to Han et al. [9]. After inoculation for $48 \mathrm{~h}$, a confocal microscope (Leica SP8, Leica Microsystems Co., Germany) was applied to observe the GFP fluorescence of the leaf discs at $488 \mathrm{~nm}$ excitation.

\section{Yeast one-hybrid assay $(\mathrm{Y} 1 \mathrm{H})$}

In order to test the protein-DNA interaction of AchnbZIP29, AchnMYB70 and AchnKCS promoter, Y1H was carried out. And according to the Matchmaker ${ }^{\circledR}$ Gold Yeast One-Hybrid Library Screening System (Cat. No. 630491 , TaKaRa®Dalian, China), the $\mathrm{Y} 1 \mathrm{H}$ assays were performed. Auto-activation analysis of AchnKCS promoter was conducted at first and the minimum inhibitory concentration of aureobasidin A (AbA, a yeast toxin) was determined. The recombinant plasmid of AchnKCS-Pro-pABAi was transferred into Y1H Gold through PEG/LiAc after linearizing. The full-length regions of AchnbZIP29 and AchnMYB70 were cloned into pGADT7 vector (AD) via restriction enzyme cutting sites (EcoRl and Xhol sites, Smal and Sacl sites, respectively). Transformed Y1H Gold harboring both AchnKCS-Pro-pABAi and AchnbZIP29-pGADT7 or AchnMYB70-pGADT7 were cultured to test the interaction on SD/-Leu with AbA at $30{ }^{\circ} \mathrm{C}$ for 3 days. And $\mathrm{Y} 1 \mathrm{H}$ Gold co-transformed with p53-promoter and pGADT7-Recwere used as positive control. Y1H Gold co-transformed with AchnKCS-Pro-pABAi and empty pGADT7 was used as negative control.

\section{Dual luciferase assay}


Dual-luciferase assay was carried out to determine the trans-activation role of AchnbZIP29 and AchnMYB70 on target AchnKCS promoter. The implementation details referred to Tao, et al. [20].

The promoter sequence of AchnKCS was inserted into LUC vector (pGreen II 0800-LUC, cut by Hindll and BamH). The CDSs of AchnbZIP29 and AchnMYB70 were amplified and inserted into pGreen II $002962-$ SK vector (SK) (cut by Hindlll and BamH), respectively. And the ClonExpress II One Step Cloning Kit (C112-01, Vazyme, China) was applied to drive the connection reactions. The procedures of Agrobacterium tumefaciens transformation and the preparation of the infection buffer of Agrobacteria were according to Han et al [9]. Afterwards, the Agrobacteria culture mixtures of AchnbZIP29 or AchnMYB70 and AchnKCS promoter (v/v 10:1) were prepared to infect tobacco leaves with needleless syringes. After $72 \mathrm{~h}$ for infiltration, the Dual-Luciferase Reporter Assay System (E1910, Promega, USA) with Modulus Luminometers (Promega, USA) was performed to detect the activities of firefly luciferase (LUC) and renilla luciferase (REN). Six biological replicates were considered to determine the results.

\section{Real-time quantitative reverse transcription PCR analysis (qRT-PCR)}

The first-strand cDNA was obtained by RNA reverse transcription according to the manufacturer's instructions of PrimeScript ${ }^{\mathrm{TM}} \mathrm{RT}$ reagent Kit (Perfect Real Time, TaKaRa Bio Inc., China). The CFX96TouchTM Deep Well Sequence Detection system (Bio-Rad Laboratories, Inc. CA, USA) was applied to detect gene transcription levels with SYBR ${ }^{\circledR}$ Premix Ex Taq ${ }^{T M}$ II (TliRNaseH Plus, TaKaRa Bio Inc., China). Each gene was analyzed in triplicate and Actin was used as reference gene. The relative expression levels of genes were calculated by the $2^{-\triangle \Delta C T}$ method [21] and presented in multiples relative to the initial value without any treatment (normalized to 1 ).

\section{Statistical analysis}

Data represented the mean value minus or plus standard deviation ( \pm SD). SPSS software (version 20.0) was used to analyze the significance of the differences by Least significant difference (LSD) test, and Origin 9.0 software was used to draw figures. The difference was considered to be statistical significance when the $p$ value was less than 0.05 and extremely significant difference when less than 0.01 , and expressed with different letters or " $\star$ ", " $\star \star *$ in figures.

\section{Results}

\section{Analysis of AchnKCS promoter sequence}

Based on the total DNA template of kiwifruit, a 709 bp sequence of AchnKCSpromoter was successfully amplified by the primer of AchnKCS-Pro-F/R in Supplementary Table 1. The sequence analysis through PlantCARE software (http://bioinformatics.psb.ugent.be/webtools/plantcare/html) showed that cisacting elements of ABRE (ABA responsive element), G-box, MBS and MRE were contained (Table 1). ABRE was considered to be specifically recognized by bZIP transcription factors and involved in ABA response, while G-box was supposed as coupling of ABRE $[22,23]$. MBS and MRE were the binding sites of MYB transcription factors [24]. 
Table 1 Bioinformatic analysis of AchnKCS promoter

\begin{tabular}{|c|c|c|c|}
\hline Element & Description & $\begin{array}{l}\text { Sequence } \\
\left(5^{\prime}-3^{\prime}\right)\end{array}$ & Position \\
\hline ABRE & $\begin{array}{l}\text { cis-acting element involved in the abscisic acid } \\
\text { responsiveness }\end{array}$ & $\underline{\text { TACGTG }}$ & $-1025(+)$ \\
\hline ABRE & $\begin{array}{l}\text { cis-acting element involved in the abscisic acid } \\
\text { responsiveness }\end{array}$ & $\underline{\text { TACGTG }}$ & $-1498(-)$ \\
\hline G-Box & $\begin{array}{l}\text { cis-acting regulatory element involved in light } \\
\text { responsiveness }\end{array}$ & $\underline{\text { CACGTT }}$ & $-1587(-)$ \\
\hline G-Box & $\begin{array}{l}\text { cis-acting regulatory element involved in light } \\
\text { responsiveness }\end{array}$ & $\underline{\text { CACGTC }}$ & $-961(-)$ \\
\hline MBS & MYB binding site involved in drought-inducibility & $\underline{\text { CAACTG }}$ & $-1203(-)$ \\
\hline MRE & MYB binding site involved in light responsiveness & AACCTAA & $-999(+)$ \\
\hline
\end{tabular}

Note: Positon represents the cis-acting element is counted from the position of ATG.

\section{Amino acid sequence homology}

Through the promoter sequence analysis and bioinformatics searching, a bZIP (Achn340751) and an MYB (Achn117821) transcription factor were inferred to be downstream responses of ABA signaling and be associated with suberin biosynthesis based on the involvement of their close homologs in ABA responding and mechanical stress [25-30]. Using cDNA as template, the CDS of Achn340751 and Achn117821 were cloned. Furthermore, the BLAST online software was used to analyze the sequence homology from the NCBI database. Based on its homology with Arabidopsis transcription factors presented as phylogenetic tree by means of DNAMAN8 and MEGA7 software in Figure 2, they were temporarily designated as AchnbZIP29 and AchnMYB70. And it showed that AchnbZIP29 and AchnMYB70 respectively belonged to Group I of bZIP transcription factors and R2R3-MYB 22 subgroup, which involved in the regulation of fatty acid biosynthesis [31-33].

\section{Subcellular localization}

In order to speculate the functional mechanism, the subcellular localization of both transcription factors was determined by observing the fluorescence signal of GFP based on the fusion expression vectors of the reporter gene GFP with AchnbZIP29 or AchnMYB70. The result displayed that compared with the green fluorescence appearing in the whole cell of the hollow GFP vector, the GFP green fluorescence signal of the fusion expression vector with the AchnbZIP29 or AchnMYB70 appeared specifically in the nucleus (Figure 3). It indicated that AchnbZIP29 and AchnMYB70 were located in the nucleus, conforming their functional characteristics of regulating gene transcription.

\section{Interaction between AchnbZIP29, AchnMYB70 and AchnKCS promoter}


$\mathrm{Y} 1 \mathrm{H}$ was carried out to investigate whether AchnbZIP29 and AchnMYB70 can interact with AchnKCS promoter. Firstly, the self-activation test showed that the yeast transformed with AchnKCS-Pro-pABAi cannot grow on the medium containing $100 \mathrm{ng} \mathrm{mL}^{-1} \mathrm{AbA}$ (Figure 4). Subsequently, $\mathrm{Y} 1 \mathrm{H}$ displayed that the positive control strain (AD-Rec-p53 + p53 promoter, not shown) and Y1HGold transformed with AchnbZIP29 + AchnKCSPro, and AchnMYB70 + AchnKCSPro can grow in the medium with $100 \mathrm{ng} \mathrm{mL}^{-}$

${ }^{1} \mathrm{AbA}$ and no leucine (-Leu) (Figure 4), which verified the interaction of individually AchnbZIP29 and AchnMYB70 with AchnKCS promoter.

Besides, in order to further clarify the regulatory effect of AchnbZIP29 and AchnMYB70 on AchnKCS, a dual luciferase assay was applied. It presented that AchnMYB70 can significantly enhance the transcriptional activity of AchnKCS promoter, and the ratio of LUC/REN was 2.32 times that of the control (SK) (Figure 5). In contrast, AchnbZIP29 negatively regulated the transcriptional activity of AchnKCS promoter, and its LUC/REN ratio was only 0.44 that of SK (Figure 5).

\section{Effect of exogenous ABA on the transcription levels of AchnbZIP29 and AchnMYB70}

The relative transcription levels of AchnbZIP29 and AchnMYB70 in ABA-stimulated suberizing tissue of kiwifruit were analyzed by qRT-PCR. As shown in Figure 6, the transcription level of AchnbZIP29 was reduced by exogenous $A B A$ and decreased to 0.45 of the initial value (normalized to 1 ) on the third day after treatment. On the contrary, the transcription level of $A c h n M Y B 70$ was significantly up-regulated by ABA. From the second day after treatment, the transcription level of AchnMYB70 in the suberizing tissue increased significantly and reached the maximum abundance on the third day, which was 2.1 times of the initial control value. The difference in relative transcript abundance induced by ABA further illustrated that AchnbZIP29 and AchnMYB70 were ABA signal-responsive transcription factors.

\section{Discussion}

Abscisic acid ( $A B A)$ is a stress resistance hormone in plant, which is involved in a variety of biotic and abiotic stresses $[34,35]$. Relevant studies in recent years have shown that ABA promoted suberin accumulation in Arabidopsis root [5,36], potato tuber [4,37], tomato [6, 38] and kiwifruit [1]. ABA treatment could induce VLCFAs accumulation during wound suberization $[4,39,40]$. KCSs are the ratelimiting enzymes in the chain elongation of fatty acids in VLCFAs biosynthesis ${ }^{41}$ and significantly induced by ABA [9].

The promoter sequence of ABA-responsive genes generally has a conserved cis-acting element, namely ABA-responsive element (ABRE; PyACGTGG/TC) [42, 43]. Transcription factors of bZIP family in plant could interact with cis-acting elements containing ACGT sequence to participate in ABA signaling. In Arabidopsis, it has identified eighty bZIP transcription factors, which are divided into 13 groups based on the similarity of their basic regions and other conserved motifs [44]. It was reported that AchnABF2 and AchnbZIP12 in Group A responding to ABA activated the transcription of AchnFHT and AchnKCS involved in suberin biosynthesis, respectively $[9,17]$. In this work, AchnbZIP29 was cloned from kiwifruit and the 
analysis of amino acid sequence showed that it was classified into Group I. The bZIPs of Group I in Arabidopsis were likely to be involved in the development of vascular tissue and cell wall [45].

AchnbZIP29 presented high homology with AtbZIP29. Related research revealed that ABA decreased the expression of AtbZIP29 in guard cells [46]. Similarly, the transcription level of AchnbZIP29 was downregulated by ABA during wound suberization in this work. It was also speculated that AtbZIP29 regulated the expression of CYP707A3 and CYP707A1 which were two key enzymes involved in ABA catabolism [29]. Accordingly, it was inferred that AchnbZIP29 negatively correlated with the expression of ABAresponsive genes and it was likely to participate in the regulation of wound suberization on the cell wall, but its target gene was possible not only AchnKCS.

However, a kind of cis-acting element was not sufficient for regulating the transcription of ABA-responsive genes. The interaction between AREB (ABRE binding proteins) and ABRE required the participation of coupling elements [47]. Considered as a coupling element of ABRE motif, the G-box element was reported to play roles in regulating gene expression under various environmental stresses [48]. Certain bZIP transcription factors contained motifs that recognized and bound to G-box element [22, 48]. In this work, the cloned AchnKCS promoter region contained not only two ABRE elements, but also two G-box elements. It allowed us to further determine that bZIP transcription factors played an important regulatory role in the ABA-promoted suberization.

MYB transcription factor family has a wide range of function diversity, including the regulation of suberin biosynthesis [10,11, 13]. In this work, AchnMYB70 was found to activate the AchnKCS promoter and positively regulate the AchnKCS transcription. Most MYB proteins bound to one or more cis-acting elements (MBS/MRE) with the conserved sequence of CNGTT(A/G) or $C(G / T) T(A / T) G T T(A / G)$ [24]. It showed that AchnMYB70 had high homology with AtMYB70, AtMYB73 and AtMYB44, which were involved in secondary metabolism and resisting biotic and abiotic stress in Arabidopsis [25, 49, 50]. The lipid content in seeds and leaves of transgenic Arabidopsis overexpressing the GmMYB73 (Glycine max) gene was significantly increased [32]. It was also reported that osmotic stress induced the transcription of AtMYB30 and AtMYB4, which was associated with the FAE complex and contributed to the synthesis of VLCFAs [51]. In addition, AchnMYB107 and AchnMYB41 were induced by exogenous ABA during wound suberization of kiwifruit and were demonstrated to activate the transcription of AchnFHT, AchnFAR and AchnCYP86A1 that were involved in suberin biosynthesis $[17,52,53]$. In this study, the transcription level of $A c h n M Y B 70$ was also up-regulated by exogenous ABA treatment and was proved to have an activating effect on AchnKCS transcription during wound suberization of kiwifruit.

The transcription of a gene may be comprehensively regulated by multiple transcription factors, and the interaction between transcription factors may jointly play a role in the transcriptional regulation of the target genes. In this work, any interaction or other cooperative regulation between the transcription factors that can interact with the AchnKCS promoter, including AchnbZIP29, AchnMYB70 and AchnbZIP12 we reported previously, still needed to be further studied.

\section{Conclusions}


In conclusion, the present work explored a potential regulatory pathway of ABA on AchnKCS involved in suberin biosynthesis (Fig. 7). AchnKCS promoter was activated by the interaction with AchnMYB70 but suppressed by the interaction with AchnbZIP29. The transcription level of AchnMYB70 was induced by ABA, but AchnbZIP29 expression was reduced by ABA. Therefore, ABA played a key role in the transcriptional activation of AchnKCS by up-regulating AchnMYB70 expression and down-regulating AchnbZIP29 expression.

\section{Abbreviations}

ABA: Abscisic acid, AbA: Aureobasidin A, ABRE: ABA responsive element, AREB: ABRE binding proteins, CDS: Coding sequence, CTAB: Cetyltrimethylammonium bromide, FAE: Fatty acid elongation enzyme complex, GFP: Green fluorescent protein, KCS: $\beta$-ketoacyl-coenzyme A synthase, LUC: Firefly luciferase, NCBI: National Center of Biotechnology Information, qRT-PCR: Real-time quantitative reverse transcription PCR, REN: Renilla luciferase, VLCFAs: Very long chain fatty acids, Y1H: Yeast one-hybrid.

\section{Declarations}

\section{Acknowledgment}

Not applicable.

\section{Authors' contributions}

Conception and design: LC Mao and XY Han, Analysis and interpretation of the data: XY Han, XP Wei and WJ Lu, Drafting of the article: XY Han and XP Wei, Critical revision of the article: $Q$ Wu and LC Mao. Final approval of the article: LC Mao, XY Han, XP Wei, WJ Lu and Q Wu.

\section{Funding}

This research was supported by the National Natural Science Foundation of China (31372113) and the General program of the Education Department of Zhejiang Province (Y202044419).

\section{Availability of data and materials}

All data generated or analyzed during this study are included in this article (and its supplementary information files) or are available from the corresponding author on reasonable request.

\section{Ethics approval and consent to participate}

Not applicable.

\section{Consent for publication}

Not applicable. 


\section{Competing interests}

The authors declare that they have no competing interests.

\section{References}

1. Han XY, Mao LC, Wei XP, Lu WJ. Stimulatory involvement of abscisic acid in wound suberization of postharvest kiwifruit. Scientia Horticulturae. 2017,224:244-250.

2. Franke RB, Dombrink I, Schreiber L. Suberin goes genomics: use of a short living plant to investigate a long lasting polymer. Front Plant Sci. 2012,3:1-8.

3. Pollard M, Beisson F, Li Y, Ohlrogge JB. Building lipid barriers: biosynthesis of cutin and suberin. Trends in Plant Science. 2008,13:236-246.

4. Lulai EC, Suttle JC, Pederson SM. Regulatory involvement of abscisic acid in potato tuber woundhealing. Journal of Experimental Botany. 2008,59:1175-1186.

5. Barberon M, Vermeer JEM, De Bellis D, Wang P, Naseer S, Andersen TG, Humbel BM, Nawrath C, Takano J, Salt DE. Adaptation of root function by nutrient-induced plasticity of endodermal differentiation. Cell. 2016,164:447-459.

6. Tao X, Mao L, Li J, Chen J, Lu W, Huang S. Abscisic acid mediates wound-healing in harvested tomato fruit. Postharvest Biology and Technology. 2016,118:128-133.

7. Han X-y, Mao L-c, Lu W-j, Tao X-y, Wei X-p, Luo Z-s. Abscisic acid induces differential expression of genes involved in wound suberization in postharvest tomato fruit. Journal of Integrative Agriculture. 2018,17:2670-2682.

8. Capote T, Barbosa P, Usié A, Ramos AM, Inácio V, Ordás R, Gonçalves S, Morais-Cecílio L. ChIP-Seq reveals that QsMYB1 directly targets genes involved in lignin and suberin biosynthesis pathways in cork oak (Quercus suber). BMC Plant Biology. 2018,18:1-19.

9. Han X, Mao L, Lu W, Wei X, Luo Z. Positive Regulation of the Transcription of AchnKCS by a bZIP Transcription Factor in Response to ABA-Stimulated Suberization of Kiwifruit. Journal of Agricultural and Food Chemistry. 2019,67.

10. Kosma DK, Murmu J, Razeq FM, Santos P, Bourgault R, Molina I, Rowland O. AtMYB41 activates ectopic suberin synthesis and assembly in multiple plant species and cell types. The Plant Journal. 2014,80:216-229.

11. Lashbrooke J, Cohen H, Levy-Samocha D, Tzfadia O, Panizel I, Zeisler V, Massalha H, Stern A, Trainotti L, Schreiber L. MYB107 and MYB9 homologs regulate suberin deposition in angiosperms. The Plant Cell. 2016,28:2097-2116.

12. Gou M, Hou G, Yang H, Zhang X, Cai Y, Kai G, Liu C-J. The MYB107 transcription factor positively regulates suberin biosynthesis. Plant Physiology. 2017,173:1045-1058.

13. Legay S, Guerriero G, André C, Guignard C, Cocco E, Charton S, Boutry M, Rowland O, Hausman JF. MdMyb93 is a regulator of suberin deposition in russeted apple fruit skins. New Phytol. 
2016,212:977-991.

14. Legay S, Guerriero G, Deleruelle A, Lateur M, Evers D, André CM, Hausman J-F. Apple russeting as seen through the RNA-seq lens: strong alterations in the exocarp cell wall. Plant Molecular Biology. 2015,88:21-40.

15. To A, Joubès J, Thueux J, Kazaz S, Lepiniec L, Baud S. AtMYB92 enhances fatty acid synthesis and suberin deposition in leaves of Nicotiana benthamiana. The Plant Journal 2020, 103, 660-676.

16. Cohen H, Fedyuk V, Wang C, Wu S, Aharoni A. SUBERMAN regulates developmental suberization of the Arabidopsis root endodermis. The Plant Journal. 2020,102:431-447.

17. Wei X, Lu W, Mao L, Han X, Wei X, Zhao X, Xia M, Xu C. ABF2 and MYB transcription factors regulate feruloyl transferase FHT involved in ABA-mediated wound suberization of kiwifruit. Journal of Experimental Botany. 2020,71:305-317.

18. Jaakola L, Pirttila AM, Halonen M, Hohtola A. Isolation of high quality RNA from bilberry ( Vaccinium myrtillus L.) fruit. Molecular Biotechnology. 2001,19:201-203.

19. Porebski S, Bailey LG, Baum BR. Modification of a CTAB DNA extraction protocol for plants containing high polysaccharide and polyphenol components. Plant Mol. Biol. Rep. 1997,15:8-15.

20. Tao RY, Bai SL, Ni JB, Yang QS, Zhao Y, Teng YW. The blue light signal transduction pathway is involved in anthocyanin accumulation in 'Red Zaosu' pear. Planta. 2018,248:37-48.

21. Livak KJ, Schmittgen TD. Analysis of relative gene expression data using real-time quantitative PCR and the $2^{-\triangle \triangle C T}$ method. Methods. 2001,25:402-408.

22. Baena-Gonzalez E, Rolland F, Thevelein JM, Sheen J. A central integrator of transcription networks in plant stress and energy signalling. Nature. 2007,448:938-U10.

23. Iglesias-Fernandez R, Barrero-Sicilia C, Carrillo-Barral N, Onate-Sanchez L, Carbonero P. Arabidopsis thaliana bZIP44: a transcription factor affecting seed germination and expression of the mannanase-encoding gene AtMAN7. Plant J. 2013,74:767-780.

24. Romero I, Fuertes A, Benito MJ, Malpica JM, Leyva A, Paz-Ares J. More than 80 R2R3-MYB regulatory genes in the genome of Arabidopsis thaliana. Plant J. 1998,14:273-284.

25. Jung C, Seo JS, Han SW, Koo YJ, Kim CH, Song SI, Nahm BH, Do Choi Y, Cheong J-J. Overexpression of AtMYB44 enhances stomatal closure to confer abiotic stress tolerance in transgenic Arabidopsis. Plant Physiology. 2008,146:623-635.

26. Persak H, Pitzschke A. Tight Interconnection and Multi-Level Control of Arabidopsis MYB44 in MAPK Cascade Signalling. Plos One. 2013,8.

27. Tsugama D, Liu S, Fujino K, Takano T. Possible inhibition of Arabidopsis VIP1-mediated mechanosensory signaling by streptomycin. Plant Signaling \& Behavior. 2018,13:e1521236.

28. Tu M, Wang X, Zhu Y, Wang D, Zhang X, Cui Y, Li Y, Gao M, Li Z, Wang Y. VlbZIP30 of grapevine functions in dehydration tolerance via the abscisic acid core signaling pathway. Horticulture Research. 2018,5:1-15. 
29. Van Leene J, Blomme J, Kulkarni SR, Cannoot B, De Winne N, Eeckhout D, Persiau G, Van De Slijke E, Vercruysse L, Bossche RV, Heyndrickx KS, Vanneste S, Goossens A, Gevaert K, Vandepoele K, Gonzalez N, Inze D, De Jaeger G. Functional characterization of the Arabidopsis transcription factor bZIP29 reveals its role in leaf and root development. Journal of Experimental Botany. 2016,67:58255840.

30. Zhao Y, Xing L, Wang XG, Hou YJ, Gao JH, Wang PC, Duan CG, Zhu XH, Zhu JK. The ABA Receptor PYL8 Promotes Lateral Root Growth by Enhancing MYB77-Dependent Transcription of AuxinResponsive Genes. Sci. Signal. 2014,7:11.

31. Gibalová A, Steinbachová L, Hafidh S, Bláhová V, Gadiou Z, Michailidis C, Müller K, Pleskot R, Dupl'áková $\mathrm{N}$, Honys $\mathrm{D}$. Characterization of pollen-expressed bZIP protein interactions and the role of ATbZIP18 in the male gametophyte. Plant Reproduction. 2017,30:1-17.

32. Liu YF, Li QT, Lu X, Song QX, Lam SM, Zhang WK, Ma B, Lin Q, Man WQ, Du WG, Shui GH, Chen SY, Zhang JS. Soybean GmMYB73 promotes lipid accumulation in transgenic plants. BMC Plant Biology. 2014,14:16.

33. Yu Y, Qian Y, Jiang M, Xu J, Yang J, Zhang T, Gou L, Pi E. Regulation Mechanisms of Plant Basic Leucine Zippers to Various Abiotic Stresses. Frontiers in Plant Science. 2020,11:1258.

34. Himmelbach A, Yang Y, Grill E. Relay and control of abscisic acid signaling. Current Opinion in Plant Biology. 2003,6:470-479.

35. Leng P, Yuan B, Guo YD. The role of abscisic acid in fruit ripening and responses to abiotic stress. Journal of Experimental Botany. 2014,65:4577-4588.

36. Tao Q, Jupa R, Liu Y, Luo J, Li J, Kováč J, Li B, Li Q, Wu K, Liang Y. Abscisic acid-mediated modifications of radial apoplastic transport pathway play a key role in cadmium uptake in hyperaccumulator Sedum alfredii. Plant, Cell \& Environment. 2019,42:1425-1440.

37. Kumar GM, Lulai EC, Suttle JC, Knowles NR. Age-induced loss of wound-healing ability in potato tubers is partly regulated by ABA. Planta. 2010,232:1433-1445.

38. Leide J, Hildebrandt U, Hartung W, Riederer M, Vogg G. Abscisic acid mediates the formation of a suberized stem scar tissue in tomato fruits. New Phytol. 2012,194:402-415.

39. Han XY, Lu WJ, Wei XP, Li L, Mao LC, Zhao YY. Proteomics analysis to understand the ABA stimulation of wound suberization in kiwifruit. J. Proteomics. 2018,173:42-51.

40. Lulai EC, Neubauer JD. Wound-induced suberization genes are differentially expressed, spatially and temporally, during closing layer and wound periderm formation. Postharvest Biology and Technology. 2014,90:24-33.

41. Paul S, Gable K, Beaudoin F, Cahoon E, Jaworski J, Napier JA, Dunn TM. Members of the Arabidopsis FAE1-like 3-ketoacyl-CoA synthase gene family substitute for the Elop proteins of Saccharomyces cerevisiae. J. Biol. Chem. 2006,281:9018-9029.

42. Hattori T, Totsuka M, Hobo T, Kagaya Y, Yamamoto-Toyoda A. Experimentally determined sequence requirement of ACGT-containing abscisic acid response element. Plant Cell Physiol. 2002,43:136140.

Page 12/19 
43. Zhang WX, Ruan JH, Ho TD, You Y, Yu TT, Quatrano RS. Cis-regulatory element based targeted gene finding: genome-wide identification of abscisic acid- and abiotic stress-responsive genes in Arabidopsis thaliana. Bioinformatics. 2005,21:3074-3081.

44. Jakoby M, Weisshaar B, Droge-Laser W, Vicente-Carbajosa J, Tiedemann J, Kroj T, Parcy F. bZIP transcription factors in Arabidopsis. Trends in Plant Science. 2002,7:106-111.

45. Fukazawa J, Sakai T, Ishida S, Yamaguchi I, Kamiya Y, Takahashi Y. Repression of shoot growth,, a bZIP transcriptional activator, regulates cell elongation by controlling the level of gibberellins. Plant Cell. 2000,12:901-915.

46. Pandey S, Wang RS, Wilson L, Li S, Zhao ZX, Gookin TE, Assmann SM, Albert R. Boolean modeling of transcriptome data reveals novel modes of heterotrimeric G-protein action. Mol. Syst. Biol. 2010,6:17.

47. Agarwal PK, Jha B. Transcription factors in plants and ABA dependent and independent abiotic stress signalling. Biol. Plant. 2010,54:201-212.

48. Menkens AE, Schindler U, Cashmore AR. The G-box: a ubiquitous regulatory DNA element in plants bound by the GBF family of bZIP proteins. Trends in Biochemical Sciences. 1995,20:506-510.

49. Shin R, Burch AY, Huppert KA, Tiwari SB, Murphy AS, Guilfoyle TJ, Schachtman DP. The Arabidopsis transcription factor MYB77 modulates auxin signal transduction. The Plant Cell. 2007,19:2440-2453.

50. Stracke R, Werber M, Weisshaar B. The R2R3-MYB gene family in Arabidopsis thaliana. Current Opinion in Plant Biology. 2001,4:447-456.

51. Raffaele S, Vailleau F, Léger A, Joubès J, Miersch 0 , Huard C, Blée E, Mongrand S, Domergue F, Roby D. A MYB transcription factor regulates very-long-chain fatty acid biosynthesis for activation of the hypersensitive cell death response in Arabidopsis. The Plant Cell. 2008,20:752-767.

52. Wei X, Mao L, Lu W, Wei X, Han X, Guan W, Yang Y, Zha M, Xu C, Luo Z. Three transcription activators of $A B A$ signaling positively regulate suberin monomer synthesis by activating cytochrome $P 450$ CYP86A1 in kiwifruit. Frontiers in Plant Science. 2020,10:1650.

53. Wei X, Mao L, Wei X, Xia M, Xu C. MYB41, MYB107, and MYC2 promote ABA-mediated primary fatty alcohol accumulation via activation of AchnFAR in wound suberization in kiwifruit. Horticulture Research. 2020,7:1-10.

\section{Figures}

\section{VLCFA biosynthesis}




Figure 1

Catalysis and substrate specificity of KCSs in the elongation steps of carbon chains involved in the synthesis of VLCFAs in Arabidopsis (Kim et al., 2013). Numbers represent the number of carbon units of VLCFAs.

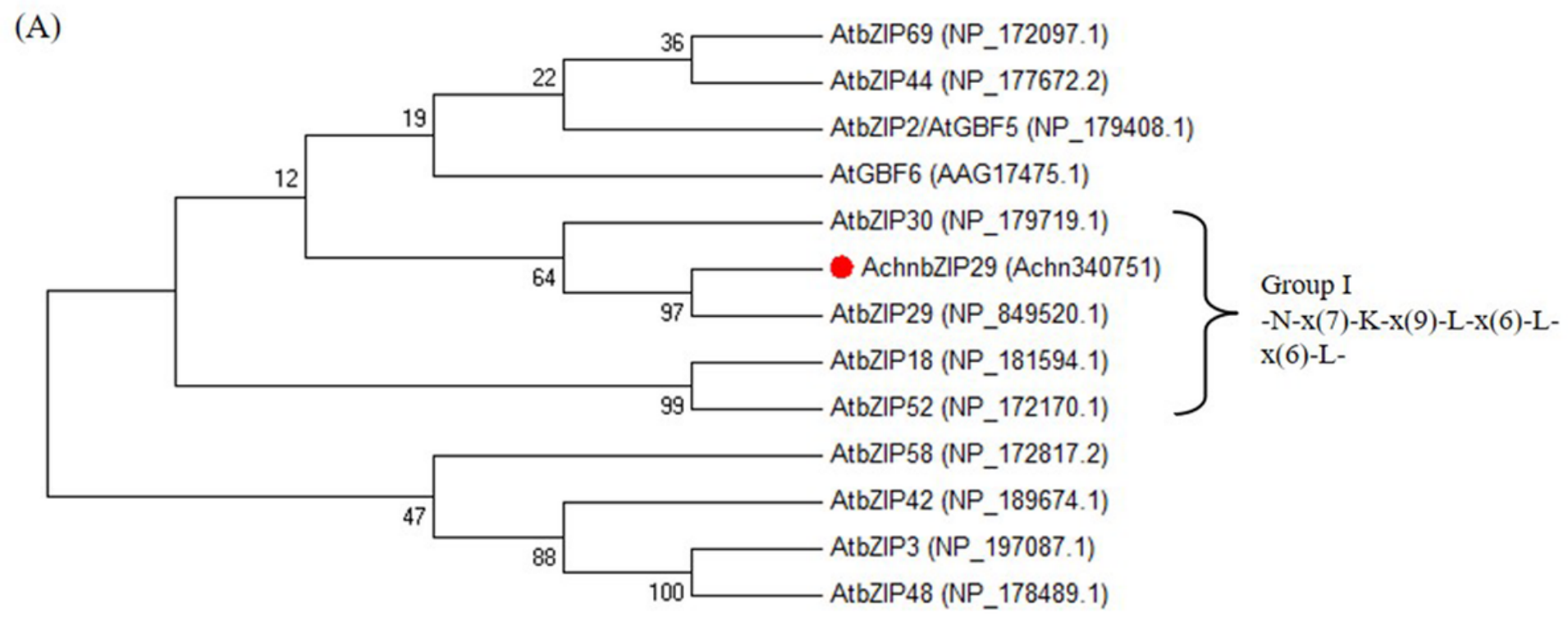

(B)

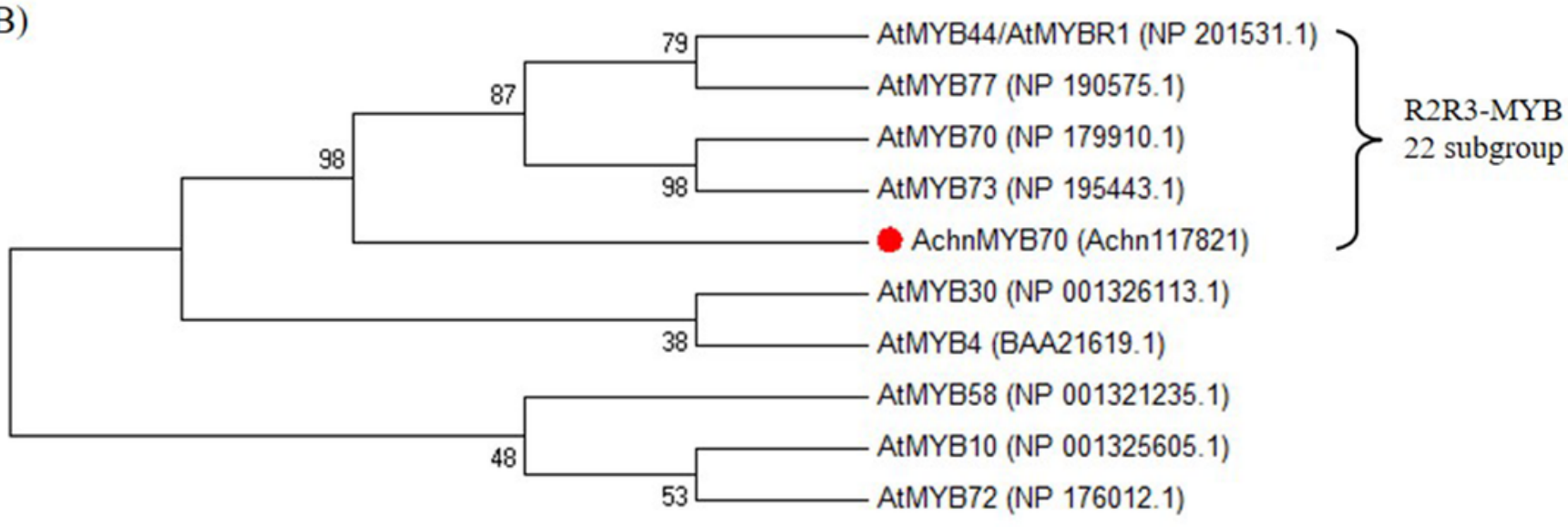

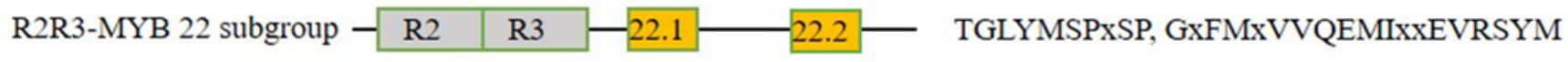

\section{Figure 2}

Amino acid sequence phylogenetic analysis of AchnbZIP29 and AchnMYB70 from kiwifruit and bZIP and MYB members from Arabidopsis. The amino acid sequences were obtained from the Cornell University kiwifruit database and NCBI database, respectively. The accession numbers were indicated in the brackets. 


\section{Empty GFP}
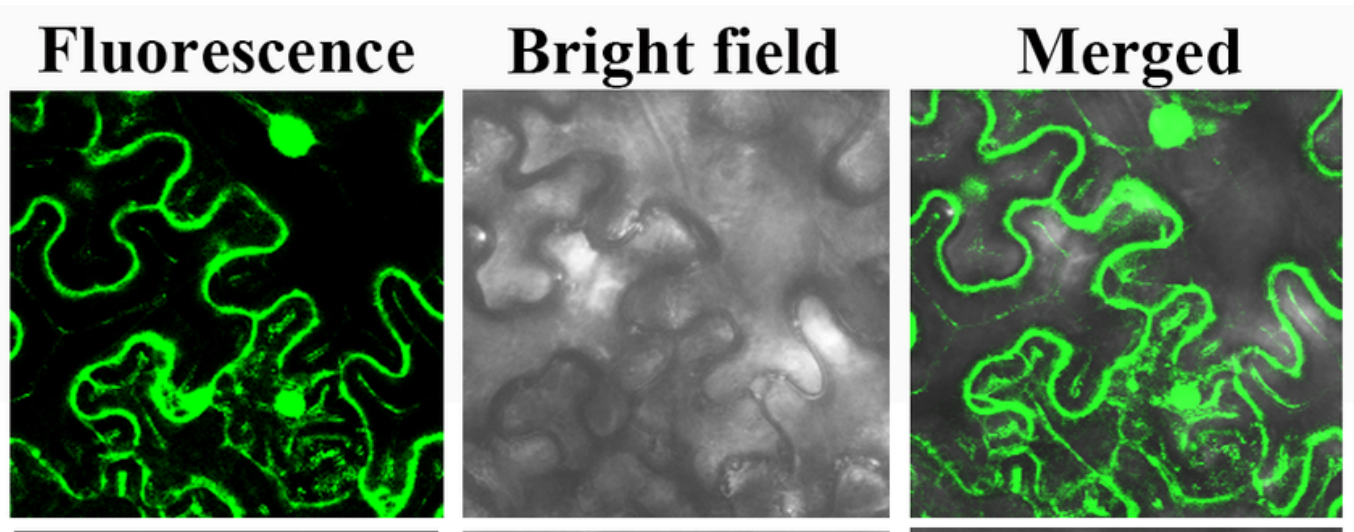

AchnbZIP29-GFP
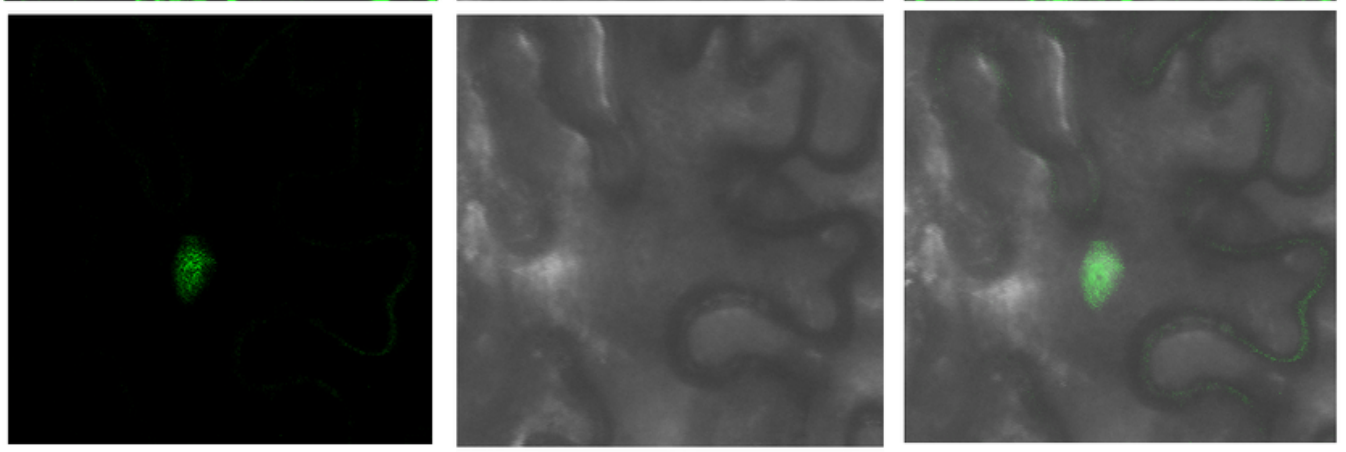

\section{AchnMYB70-GFP}
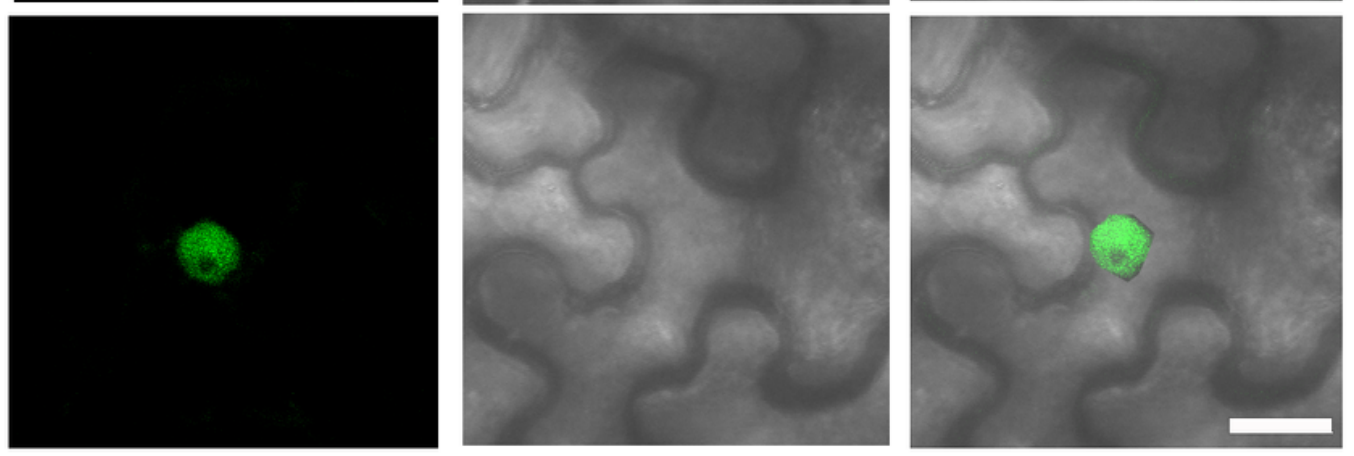

Figure 3

Subcellular localization of AchnbZIP29 and AchnMYB70 indicated by GFP green fluorescence in Nicotiana benthamiana epidermal cells. Bars $=50 \mu \mathrm{m}$. 


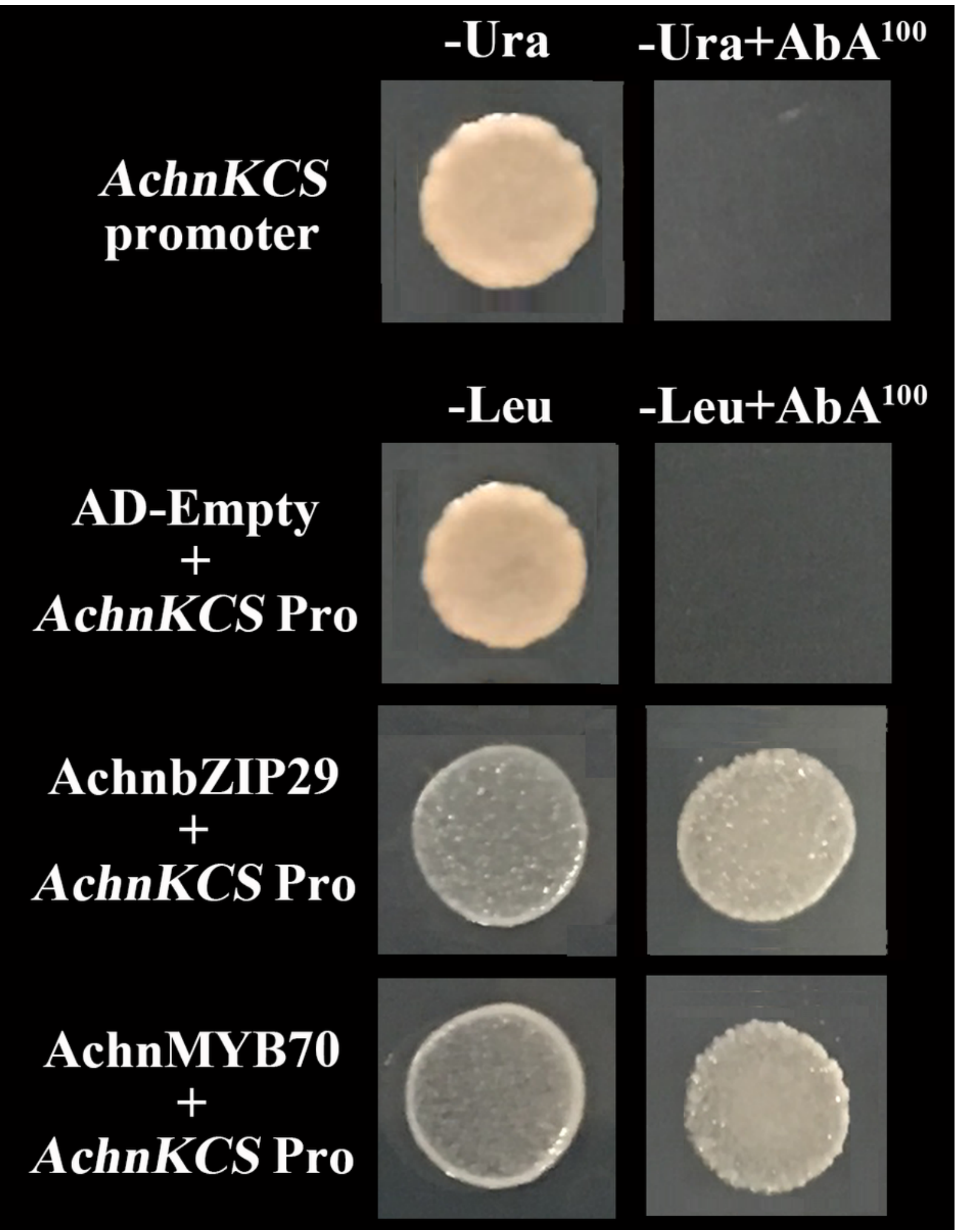

Figure 4

Yeast one-hybrid analysis on interaction between AchnbZIP29, AchnMYB70 and AchnKCS promoter 


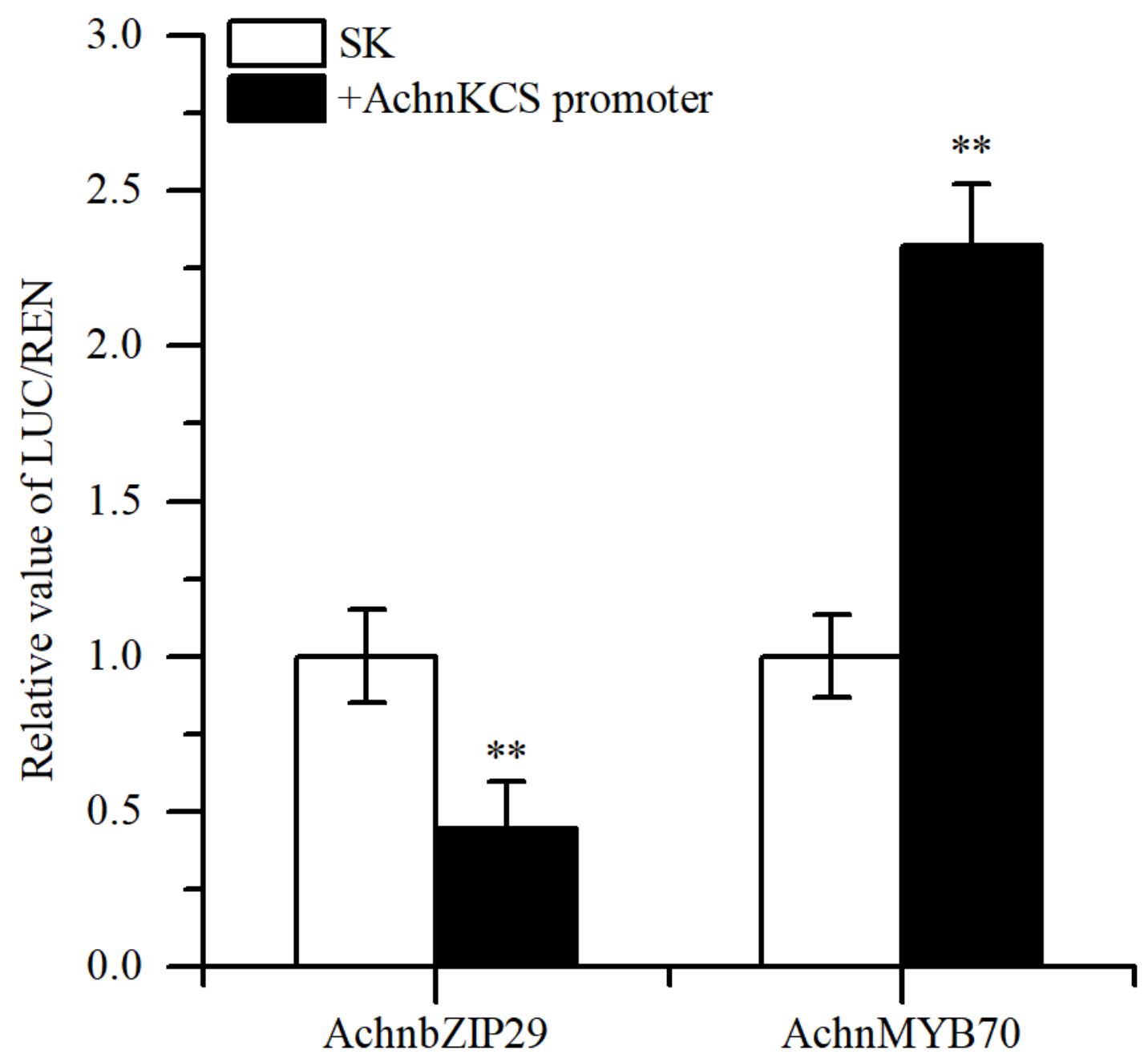

Figure 5

The transcriptional effect of AchnbZIP29 and AchnMYB70 on the promoter of AchnKCS by dualluciferase assay. The LUC/REN value for the empty vector (SK) was set as 1 . 


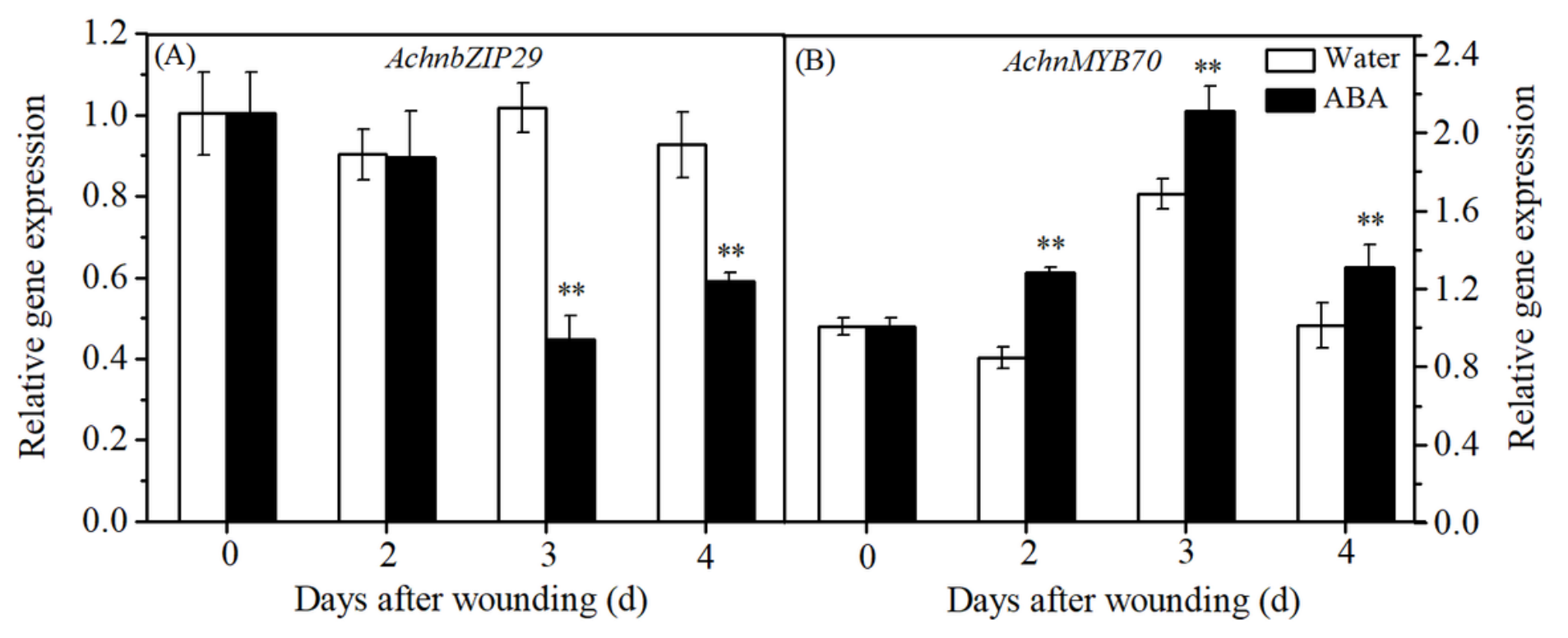

Figure 6

Relative transcription levels of AchnbZIP29 and AchnMYB70 during wound suberization of kiwifruit

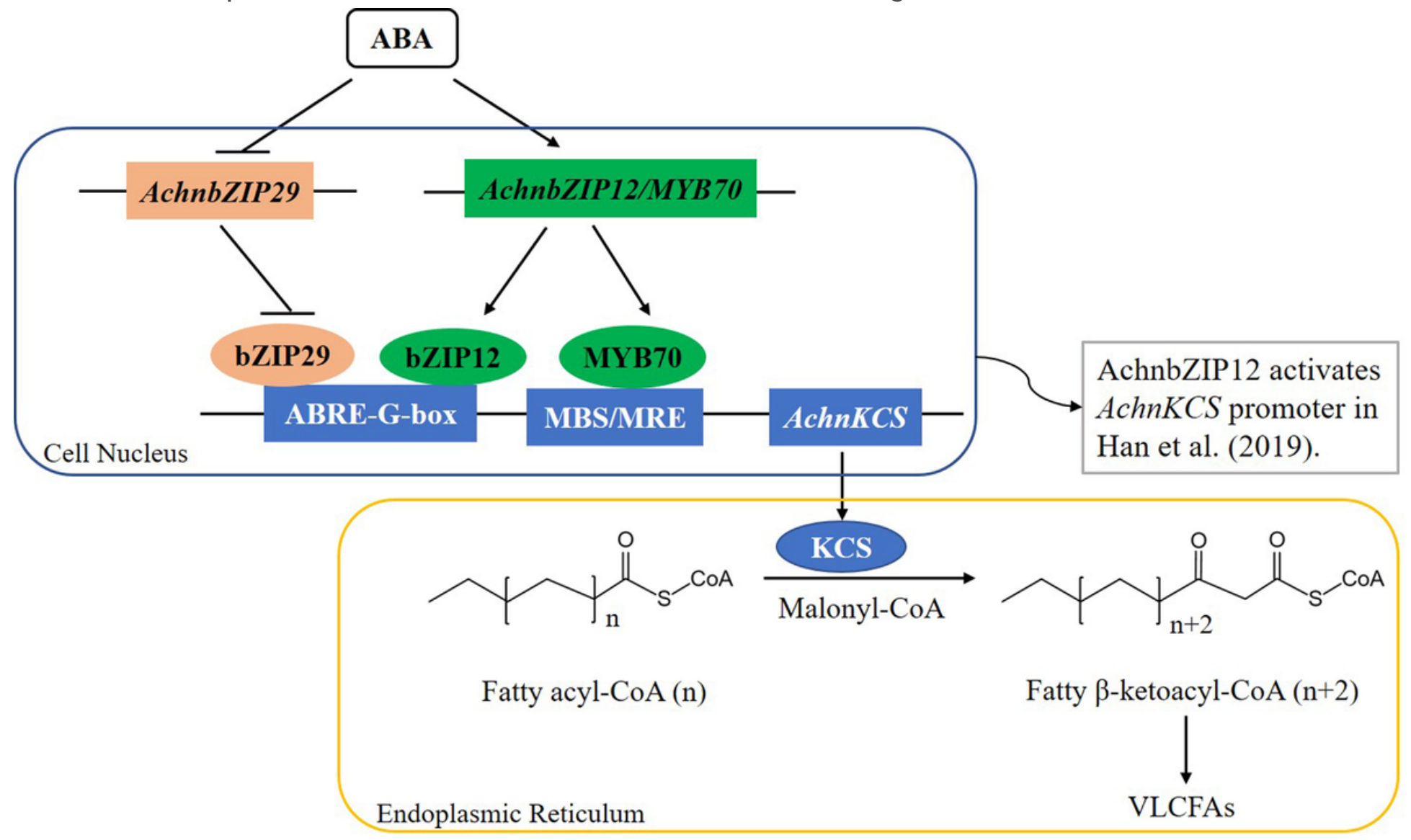

Figure 7

The model of the transcriptional regulation of AchnbZIP29, AchnMYB70 and AchnbZIP12 on AchnKCS responding to ABA during wound suberization of kiwifruit. Note: AchnbZIP12 and AchnMYB70 induced to increase AchnKCS transcription through interacting with cis-acting element (ABRE/G-box and MBS/MRE). 
The down-regulated transcription of AchnbZIP29 relieved the inhibitory effect of AchnbZIP29 on AchnKCS. And KCS as the key component of FAE complex catalyzed the chain elongation of fatty acyl$\operatorname{CoA}(\mathrm{C} n \geq \mathrm{C} 16)$ to fatty $\beta$-ketoacyl-CoA $(\mathrm{C}(\mathrm{n}+2))$, further producing VLCFAs that were precursors of suberin.

\section{Supplementary Files}

This is a list of supplementary files associated with this preprint. Click to download.

- SupplementaryTable1.docx 